\title{
Rickettsia rickettsii infection of the EA.hy 926 endothelial cell line: morphological response to infection and evidence for oxidative injury
}

\author{
Marina E. Eremeeva and David J. Silverman
}

Author for correspondence: David J. Silverman. Tel: +1 410706 7294. Fax: +1 4107063115. e-mail : dsilverm@umaryland.edu

Department of

Microbiology and

Immunology, University of

Maryland, Baltimore,

School of Medicine, 655

West Baltimore Street,

Baltimore MD 21201, USA
EA.hy 926 is a permanent human cell line that expresses highly differentiated functions characteristic of human vascular endothelium. Rickettsia rickettsii can efficiently infect and cause a cytopathic effect in EA.hy 926 cells. $R$. rickettsii produced visible lytic plaques in EA.hy 926 cells at $10 \mathrm{~d}$ postinfection (p.i.) following application of a secondary agarose overlay containing $2 \mu \mathrm{g}$ emetine $\mathrm{ml}^{-1}$ and $40 \mu \mathrm{g} \mathrm{NaF} \mathrm{ml}^{-1}$ on day 2. Rickettsial growth in EA.hy 926 cells had a similar profile to that occurring in human umbilical vein endothelial cells (HUVEC) and rickettsiae catalysed polymerization of actin tails. Intracellular multiplication of $R$. rickettsii resulted in significant changes in the internal morphology of EA.hy 926 cells, most notably extensive dilatation of the membranes of the endoplasmic reticulum and outer nuclear envelope by $72 \mathrm{~h}$ p.i. These events correlated with significant alterations in the host-cell antioxidant system, including decreased levels of intracellular reduced glutathione and glutathione peroxidase activity and increased amounts of intracellular peroxide through to $96 \mathrm{~h}$ of infection. These findings are similar to the changes described previously for $R$. rickettsii-infected HUVEC and suggest that common mechanisms associated with rickettsia-induced oxidative injury occur in the two cell lines. EA.hy 926 cells were also used to investigate the influence of the antioxidant $\alpha$-lipoic acid on rickettsial infection. Overnight pretreatment with 1-500 $\mu \mathrm{M} \alpha$-lipoic acid did not prevent cells from being destroyed following infection with rickettsiae. Supplementation of the culture medium with 1 and $10 \mu \mathrm{M} \alpha$-lipoic acid $2 \mathrm{~h}$ after rickettsial inoculation also did not provide any protective effect. However, 100, 200 and $500 \mu \mathrm{M} \alpha$-lipoic acid increased the viability of infected cells at $96 \mathrm{~h}$ to 45,51 and $70 \%$, respectively compared with $26 \%$ for untreated, infected samples. Thiol levels and glutathione peroxidase activity in treated, infected cells increased and peroxide content decreased proportionally to increasing $\alpha$-lipoic acid concentrations. Furthermore, treatment with $500 \mu \mathrm{M}$ $\alpha$-lipoic acid for $72 \mathrm{~h}$ p.i. completely prevented ultrastructural changes in infected cells. In conclusion, the permanent endothelial cell line EA.hy 926 is susceptible to injury induced by $R$. rickettsii infection. Although the cellular changes resulting from infection are not identical in all aspects to that demonstrated previously in HUVEC, the increased reproducibility and convenience of EA.hy 926 cells make them suitable for biochemical and morphological studies.

Keywords: Rickettsia rickettsii, EA.hy 926 endothelial cells, oxidative injury, $\alpha$-lipoic acid 


\section{INTRODUCTION}

Endothelial cells of small- and medium-sized vessels are the primary target cells for Rickettsia rickettsii during infection of vertebrate hosts. $R$. rickettsii, the agent of Rocky Mountain spotted fever, invades cells by induced phagocytosis, quickly escapes from the phagosome and undergoes intracellular replication that finally results in lethal injury to the host cell. The accumulated cell injury and subsequent cellular responses to rickettsial invasion are responsible for pathophysiological changes during Rocky Mountain spotted fever which include thrombosis, microhaemorrhage and vasculitis (Walker, 1988).

Human umbilical vein endothelial cells (HUVEC) comprise the same ontogenetic type of cells which rickettsiae parasitize in vivo (Silverman, 1984). Consequently, these cells are used widely as a model system for studying rickettsia-host cell interactions in vitro. However, the sequential order of host-cell events following infection with $R$. rickettsii and the mechanisms of their induction and regulation are only partially understood. Methodological limitations imposed by the growth properties of obligately intracellular bacteria like $R$. rickettsii are compounded by the peculiar physiological and biological characteristics of primary human endothelial cells. HUVEC have a very short life span in vitro and their properties can change even after a few passages (see Emeis \& Edgell, 1988). HUVEC are also intrinsically variable since they are isolated from individuals with different genetic backgrounds. Thus, they may potentially have different susceptibility to damage caused by $R$. rickettsii infection (Walker, 1988; Walker et al., 1981). Moreover, the availability of HUVEC may be constrained by medical and ethical issues and there is little control over the number and condition of cords available for preparing HUVEC. These features of primary endothelial cells limit the extent to which they can be manipulated in vitro, particularly for biochemical studies requiring significant mass to investigate the structural, functional and regulatory aspects of the bacterium-host cell interaction.

To overcome these problems, we recently introduced a permanent human endothelial cell line, EA.hy 926, to our laboratory. The EA.hy 926 cell line was obtained by fusion of HUVEC with the permanent human cell line A549 derived from a human lung carcinoma (Edgell et al., 1983). This cell line expresses highly differentiated functions that are characteristic of human vascular endothelium (Edgell et al., 1983, 1990).

The purpose of this work was to evaluate the suitability of the EA.hy 926 cell line for studying different aspects of $R$. rickettsii infection as an alternative to HUVEC. Morphological responses to rickettsial infection were studied by light and electron microscopy. Rickettsial growth, actin tail polymerization and plaque formation were investigated. Characteristics of rickettsia-induced injury and the effects of the metabolic antioxidant $\alpha$ lipoic acid also were examined. Finally, these observations were compared with studies done previously in HUVEC (Eremeeva \& Silverman, 1998).

\section{METHODS}

Cell culture. EA.hy 926 cells were kindly provided by Dr CoraJean S. Edgell (University of North Carolina, Chapell Hill, NC, USA) at the 247th passage in Dulbecco's Modified Eagle Medium (DMEM) supplemented with 4.5 g glucose $1^{-1}, 10 \%$ (v/v) foetal bovine serum, $1 \mathrm{mM}$ L-glutamine and HAT (all from Gibco-BRL). After cells were passaged three times in the same medium in our laboratory at $35^{\circ} \mathrm{C}$, they were passaged four times in the same medium supplemented with HT (GibcoBRL). Cells were cultivated in DMEM supplemented with $4.5 \mathrm{~g}$ glucose $1^{-1}, 10 \%$ foetal bovine serum and $1 \mathrm{mM} \mathrm{L}$ glutamine for the purposes of this study.

Primary human endothelial cells were isolated from veins of freshly acquired umbilical cords according to the method of Gimbrone (1976). Isolated cells were maintained in McCoy's 5A medium (Gibco-BRL) supplemented with $20 \%$ foetal bovine serum, $30 \mu \mathrm{g}$ endothelial cell growth supplement $\mathrm{ml}^{-1}$ (Upstate Biotechnology) and $50 \mu \mathrm{g}$ heparin $\mathrm{ml}^{-1}$ (Sigma) in 35 or $60 \mathrm{~mm}$ diameter Nunc tissue culture dishes precoated with $0.2 \%$ gelatin (Difco) prepared in Dulbecco's phosphatebuffered saline (PBS; Gibco-BRL).

Rickettsiae. $R$. rickettsii, strain Sheila Smith (strain VR-149), was obtained from the American Type Culture Collection (ATCC, Rockville, MD, USA). Rickettsiae were propagated in Vero cell monolayers (African green monkey kidney cells, ATCC) in DMEM supplemented with $4 \%$ foetal bovine serum and $1 \mathrm{mM}$ L-glutamine in a $5 \% \mathrm{CO}_{2}$ atmosphere as described by Santucci et al. (1992) and Silverman \& Santucci (1988). Five to six days post-infection (p.i.) the intensity of infection was examined in smears stained by the method of Gimenez (1964) and heavily infected cells were harvested using $3 \mathrm{~mm}$ diameter glass beads. Rickettsiae were purified by sonication of the cells, multiple passages of the suspension through an 18-gauge needle and differential centrifugation (Aniskovich et al., 1989). The final suspension of purified rickettsiae was prepared in SRM buffer [0.218 M sucrose, $5 \mathrm{mM}$ potassium glutamate buffer, $\mathrm{pH} 7 \cdot 0$, supplemented with $1 \%$ (w/v) Renografin-76 (E. R. Squibb \& Sons) and $5 \mathrm{mM}$ $\mathrm{MgCl}_{2}$ ], aliquoted and frozen at $-83^{\circ} \mathrm{C}$. The viable titre of purified rickettsiae was determined by plaque titration in Vero cells as described by Wike \& Burgdorfer (1972).

Infection of endothelial cells with $R$. rickettsii. The suspension of purified rickettsiae was diluted in cell culture medium and EA.hy 926 cell monolayers were inoculated at a ratio of 0.25 rickettsiae per cell in $0.3 \mathrm{ml}$ per $35 \mathrm{~mm}$ diameter dish. After rickettsiae were allowed to adhere for $2 \mathrm{~h}$ at room temperature with rocking, the volume of medium was adjusted to $2 \mathrm{ml}$ and dishes were transferred to a $35^{\circ} \mathrm{C}$ incubator and maintained in $5 \% \mathrm{CO}_{2}$. The infection was monitored at $24 \mathrm{~h}$ intervals by examination of smears stained with acridine orange and by plaquing of infected cultures on Vero cells. Cell viability was estimated by the trypan blue dye exclusion test as described by Silverman et al. (1991).

Fluorescent staining of rickettsiae and actin. Staining was performed at $72 \mathrm{~h}$ p.i. as described by Heinzen et al. (1993). The infected monolayers were fixed with $3 \%(\mathrm{w} / \mathrm{v})$ paraformaldehyde solution prepared in PBS and permeabilized by treatment with $0.5 \%$ Triton $\mathrm{X}-100$ as recommended by Clerc \& Sansonetti (1987). Rickettsiae were labelled by indirect immunofluorescence using mouse polyclonal serum prepared against Renografin-purified $R$. rickettsii (working dilution 1:200, kindly prepared by Dr G. A. Dasch, Naval Medical Research Institute, Bethesda, MD, USA) (Aniskovich et al., 1989 ; Eremeeva et al., 1993) and a 1:50 dilution of fluorescein- 
labelled goat anti-mouse $\operatorname{IgG}(\mathrm{H}+\mathrm{L}$ ) (Kirkegaard \& Perry Labs). Actin was stained with $10 \mathrm{U}$ rhodamine phalloidin $\mathrm{ml}^{-1}$ (Molecular Probes). Slides were viewed and photographed with a Zeiss fluorescence microscope using a fluorescein filter set. Under these conditions rickettsiae appear apple-green and actin fibres appear yellow-green.

Treatment of endothelial cells with $\alpha$-lipoic acid. To evaluate the effect of $\alpha$-lipoic acid (DL-6,8-thioctic acid; Sigma), $500 \mathrm{mM}$ stock solution was prepared in DMSO (Fisher Scientific) and $1-500 \mu \mathrm{M}$ were used for treatment of endothelial cells prior to or after inoculation with rickettsiae. Rickettsial infection and the effects of $\alpha$-lipoic acid were examined at $24 \mathrm{~h}$ intervals. The cell medium was aspirated, monolayers were rinsed with PBS and the dishes were processed for further analysis as described below.

Rickettsial infection was monitored microscopically after acridine orange staining of monolayers (Lauer et al., 1981) and by plaquing of infected cultures on Vero cells. Infected cells were harvested with sterile glass beads in medium, the beads and dishes washed in the same volume of $2 \times \mathrm{SRM}$ buffer and the pooled medium and washings frozen. On the day of assay, the samples were thawed and passed through an 18-gauge needle. One millilitre each of $10^{-2}-10^{-5}$ dilutions were assayed on Vero cells in triplicate in 12-well plates (Costar). The plaques were counted $7 \mathrm{~d}$ p.i. following staining with $0.3 \%$ neutral red (Gibco-BRL) in PBS for $2 \mathrm{~h}$ at $35^{\circ} \mathrm{C}$ (Wike \& Burgdorfer, 1972).

The viability of endothelial cells in monolayers during the experiment was determined by the trypan blue dye exclusion method (Silverman et al., 1991). The cells were removed from the dishes by trypsinization, pelleted by centrifugation at $200 \mathrm{~g}$ for $10 \mathrm{~min}$ and resuspended in PBS for counting.

Determination of intracellular reduced glutathione. Glutathione concentrations were determined by the method of Saville (1958) as described by Devamanoharan et al. (1994). Cell proteins were precipitated with $6.5 \%(\mathrm{w} / \mathrm{v})$ TCA followed by colorimetric measurement of reduced glutathione in extract supernatants. The colour reaction was developed after 5 min incubation with a substrate solution containing $0.2 \% N$-(1-naphthyl)ethylenediamine dihydrochloride (ICN) in distilled water. $A_{540}$ was read in a Multiscan Titretek spectrophotometer; the blank solution consisted of $6.5 \%$ TCA processed in the same manner as the supernatant fractions. The standard curve was generated from triplicate samples of serial dilutions of reduced glutathione (Sigma) and the results were expressed in nmol glutathione (mg protein $)^{-1}$. Protein concentrations were measured by the method of Smith et al. (1985) using the BCA protein assay reagent (Pierce).

Determination of glutathione peroxidase activity. Glutathione peroxidase activity was measured by the method described by Paglia \& Valentine (1967) and calculated by using the extinction coefficient of NADPH $(6.22 \mathrm{mM})$ and expressed as mU enzyme (mg protein $)^{-1}$, where $1 \mathrm{mU}$ glutathione peroxidase oxidizes $1 \mathrm{nmol}$ glutathione $\mathrm{min}^{-1}$ (Devamanoharan et al., 1994).

Determination of intracellular peroxide levels. Endothelial cells were assayed for intracellular peroxide by a modification of the method of Cathcart et al. (1983). Peroxide levels were expressed as fluorescence units (mg protein $)^{-1}$.

Transmission electron microscopy. At $72 \mathrm{~h}$ p.i., the culture medium was decanted and the cells were washed once with PBS. The monolayers were fixed overnight in situ at $4^{\circ} \mathrm{C}$ in a $2 \%(\mathrm{v} / \mathrm{v})$ glutaraldehyde solution prepared in $0.1 \mathrm{M}$ sodium cacodylate buffer, $\mathrm{pH} 7 \cdot 3$, as described by Silverman (1984).
Following fixation, the cells were washed three times in cacodylate buffer, post-fixed in $1 \%(\mathrm{w} / \mathrm{v})$ osmium tetroxide for $1 \mathrm{~h}$ at room temperature, dehydrated in an ascending ethanol series and embedded in PolyBed 812 (Polysciences). Ultrathin sections were collected on carbon-coated collodion copper grids, stained with uranyl acetate and lead citrate and viewed in a JEOL 1200 EX electron microscope operating at $60 \mathrm{kV}$.

Plaque assay in the EA.hy 926 cell line. A suspension of $R$. rickettsii containing 7.01 $\times 10^{8}$ Vero cell plaque-forming units (p.f.u.) $\mathrm{ml}^{-1}$ was titrated in EA.hy 926 cells. One millilitre of serial tenfold dilutions of rickettsial suspension was inoculated in triplicate into 12-well plates (Costar) and rickettsiae were allowed to adhere for $2 \mathrm{~h}$ at $35^{\circ} \mathrm{C}$. The inoculum was aspirated, infected cell monolayers were overlaid with $0.5 \%$ agarose prepared in culture medium and plates were cultivated at $35{ }^{\circ} \mathrm{C}$. Secondary $0.5 \%$ agarose overlay in a volume equal to the first overlay and containing $2 \mu \mathrm{g}$ emetine $\mathrm{ml}^{-1}$ and $40 \mu \mathrm{g}$ $\mathrm{NaF} \mathrm{ml^{-1 }}$ (both from Sigma) was applied on day 2 p.i. as recommended by Policastro et al. (1996). The effects of $R$. rickettsii were examined for $10 \mathrm{~d}$ p.i. following staining with $0.3 \%$ neutral red (Gibco-BRL) in PBS for $2 \mathrm{~h}$ at $35^{\circ} \mathrm{C}$.

Statistical analysis. Each experiment, except for the electron microscopy study, was repeated at least three times and three to six dishes were used for each time point and experimental variable. The mean, SD and SEM for each experimental condition were calculated. Statistical significance was assessed with Student's $t$-test $(\alpha=0.05)$ using the computer program GB-Stat 6.01 .

\section{RESULTS}

\section{Infection of the EA.hy 926 cell line with $R$. rickettsii}

Inoculation of EA.hy 926 cells with $R$. rickettsii resulted in a productive infection. $R$. rickettsii could be detected in the cytoplasm of infected cells by both acridine orange (Fig. 1) and Gimenez staining (not shown). To evaluate the kinetics of rickettsial growth in the EA.hy 926 cell line, the number of viable rickettsiae at different times was determined by plaque assay in Vero cells. Titres reached their maximum at $72 \mathrm{~h}$ p.i. $(P<0 \cdot 1)$ (Fig. 2a) and then decreased significantly between 72 and $96 \mathrm{~h}$ $(P<0.05)$, probably due to the decline in the number of viable endothelial cells available for the next cycle of

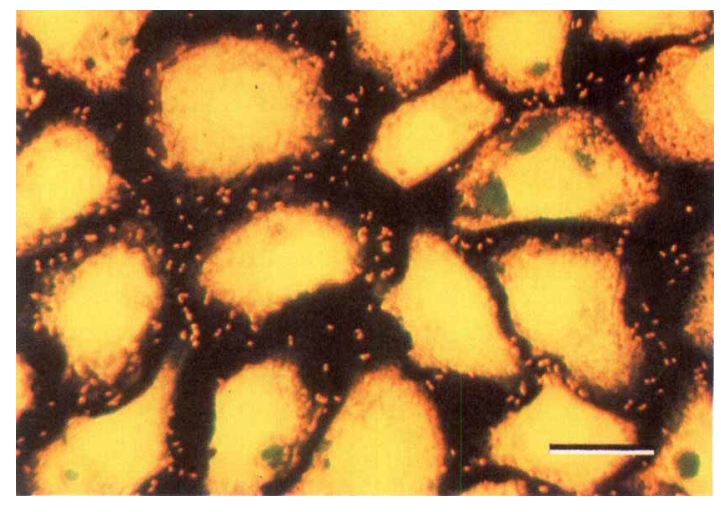

Fig. 1. Detection of $R$. rickettsii at $72 \mathrm{~h}$ p.i. in EA.hy 926 cells following acridine orange staining. Bar, $50 \mu \mathrm{m}$. 

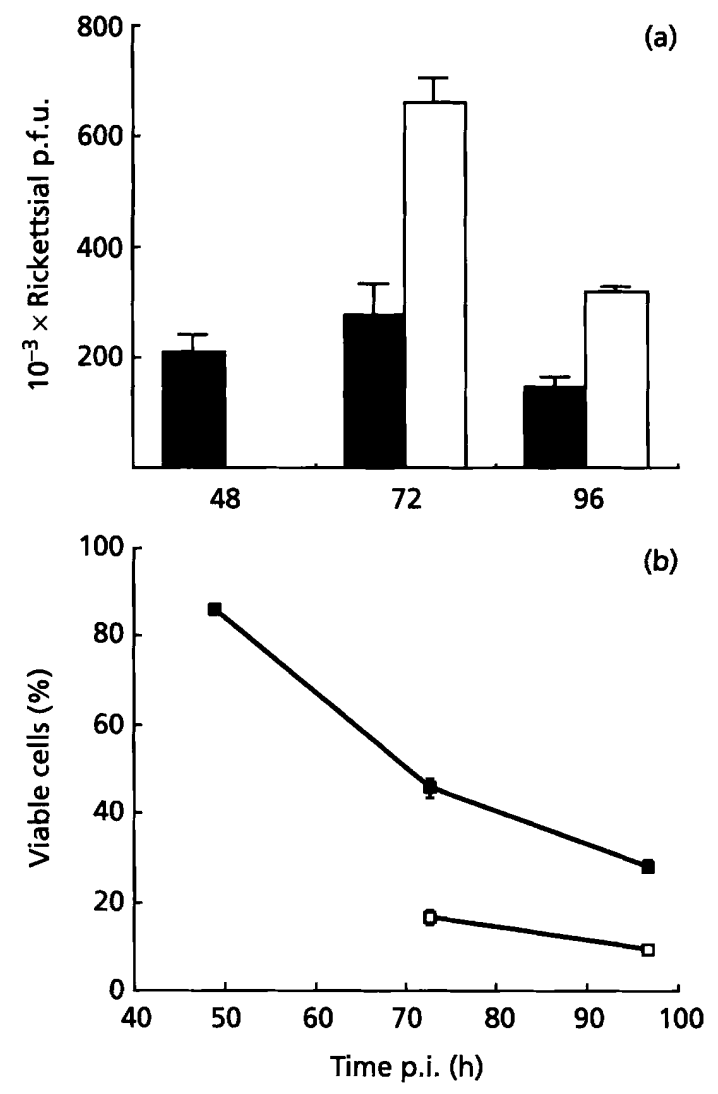

Fig. 2. Comparison of profiles of $R$. rickettsii growth and development of cytopathic effect in EA.hy 926 cells and HUVEC. (a) Recovery of viable $R$. rickettsii from infected HUVEC ( $\square$ ) and EA.hy $926(\square)$ cells as determined by plaque assay in Vero cells. The data are presented as the number of rickettsial p.f.u. (mean \pm SEM) per dish. (b) Influence of $R$. rickettsii on viability of infected HUVEC $(\square)$ and EA.hy 926 ( $\square$ ) cells. The number of viable cells was determined by the trypan blue dye exclusion test and results are expressed as the percentage of the total number of cells harvested per dish for each experimental variable. Some error bars are not seen due to the low SEM.

replication. Multiplication of $R$. rickettsii in HUVEC, used as a control in these experiments, had similar profiles (Fig. 2a). However, the total yield of viable rickettsiae was higher for $R$. rickettsii growing in HUVEC.

Multiplication of $R$. rickettsii caused a cytopathic effect in EA.hy 926 cells detected by light microscopic observation of infected monolayers beginning $72 \mathrm{~h}$ p.i. (not shown). Infected cells formed easily identifiable cytopathic foci and then detached into the cell culture medium. Increased cell detachment resulted in the appearance of holes in the infected monolayers. A significant decrease $(P<0.01)$ in the percentage of viable cells occurred by 72 and $96 \mathrm{~h}$ p.i. ( 45 and $26 \%$, respectively, as determined by the trypan blue dye exclusion test; Fig. 2b). The viability of cells in uninfected monolayers maintained under similar conditions did not change and varied from 91 to $97 \%$ over $96 \mathrm{~h}$ of observation (data not shown). The viability of

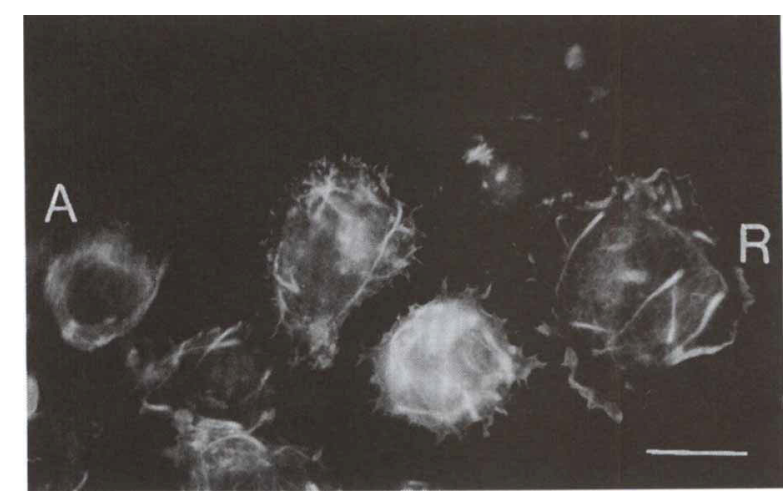

Fig. 3. Microimmunofluorescence detection of $R$. rickettsii (fluorescein staining; R) and actin (rhodamine phalloidin staining; A) in infected EA.hy 926 endothelial cells at $72 \mathrm{~h}$ p.i. Bar, $40 \mu \mathrm{m}$.

HUVEC infected with a similar inoculum of $R$. rickettsii was 15 and $8 \%$ at 72 and 96 h p.i., respectively (Fig. 2b).

\section{Microimmunofluorescence detection of rickettsiae and actin tail formation}

The cellular localization of $R$. rickettsii was examined by indirect microimmunofluorescence staining using polyclonal mouse antiserum. Apple-green rickettsiae were located in the cytoplasm of infected cells (Fig. 3). The majority of rickettsiae displayed unipolar yellowgreen actin tails of approximately $2 \mu \mathrm{m}$ thickness in close proximity to the rickettsiae. Tail lengths varied from 15 to $50 \mu \mathrm{m}$. Actin-tail-associated rickettsiae were mostly detected on the periphery of infected cells. Actin stress fibres crossing the host-cell cytoplasm were identified in only a few EA.hy 926 cells. Actin in the majority of cells stained diffusely and uniformly in the cytoplasm but some cells also had multiple circumferential actin fibres in areas adjoining the cellular membrane (Fig. 3).

\section{Oxidative changes in EA.hy 926 cells uninfected or infected with $R$. rickettsii}

Various measurements of changes in the oxidation status of EA.hy 926 cells, uninfected or infected with $R$. rickettsii, were performed at $72 \mathrm{~h}$ p.i. and compared with the respective data obtained for HUVEC (Table 1). At this time point reliable measurements of each parameter could be done for both EA.hy 926 cells and HUVEC infected at a ratio of 0.25 rickettsiae per cell. Although, similar analysis could be done with EA.hy 926 cells at $96 \mathrm{~h}$, many preparations of HUVEC could not survive $R$. rickettsii infection for $96 \mathrm{~h}$. The levels of intracellular peroxides, reduced glutathione and glutathione peroxidase in uninfected, confluent EA.hy 926 cell monolayers were significantly lower $(P<0.01)$ than the corresponding parameters in uninfected HUVEC over $72 \mathrm{~h}$ of cultivation (Table 1). R. rickettsii infection 
Table 1. Biochemical changes in endothelial cells at $72 \mathrm{~h}$ p.i. with $R$. rickettsii

Data of representative experiments are given as mean $\pm \operatorname{SEM}(n=3-4$ replicate dishes per variable).

\begin{tabular}{|c|c|c|c|}
\hline Cell type & $\begin{array}{c}\text { Intracellular peroxide } \\
{\left[\mathrm{U}(\mathrm{mg} \text { protein })^{-1}\right]}\end{array}$ & $\begin{array}{l}\text { Intracellular glutathione } \\
{\left[\text { nmol }(\text { mg protein })^{-1}\right]}\end{array}$ & $\begin{array}{l}\text { Glutathione peroxidase } \\
\text { activity }[\mathrm{mU}(\mathrm{mg} \\
\left.\text { protein })^{-1}\right]\end{array}$ \\
\hline \multicolumn{4}{|l|}{ HUVEC } \\
\hline Uninfected cells & $772 \cdot 07 \pm 53 \cdot 31$ & $121 \cdot 21 \pm 23 \cdot 78$ & $164 \cdot 72 \pm 5 \cdot 52$ \\
\hline Infected cells & $1016 \cdot 14 \pm 54 \cdot 85^{*}$ & $44 \cdot 28 \pm 3 \cdot 47^{*}$ & $89.6 \pm 7.98 *$ \\
\hline \multicolumn{4}{|l|}{ EA.hy 926} \\
\hline Uninfected cells & $435 \cdot 85 \pm 13 \cdot 76 \dagger$ & $81 \cdot 16 \pm 2 \cdot 5 \dagger$ & $112 \cdot 67 \pm 4 \cdot 48 \dagger$ \\
\hline Infected cells & $913 \cdot 37 \pm 48 \cdot 85^{*} \dagger$ & $40 \cdot 44 \pm 5 \cdot 07^{*} \ddagger$ & $55 \cdot 07 \pm 2 \cdot 18^{*} \dagger$ \\
\hline
\end{tabular}

* Infected cell value is significantly different $(P<0.05)$ from respective control cell value.

† Value is significantly different in EA.hy 926 cells $(P<0.05)$ compared to respective HUVEC value.

$\ddagger$ Value is not significantly different in EA.hy 926 cells compared to respective HUVEC value.

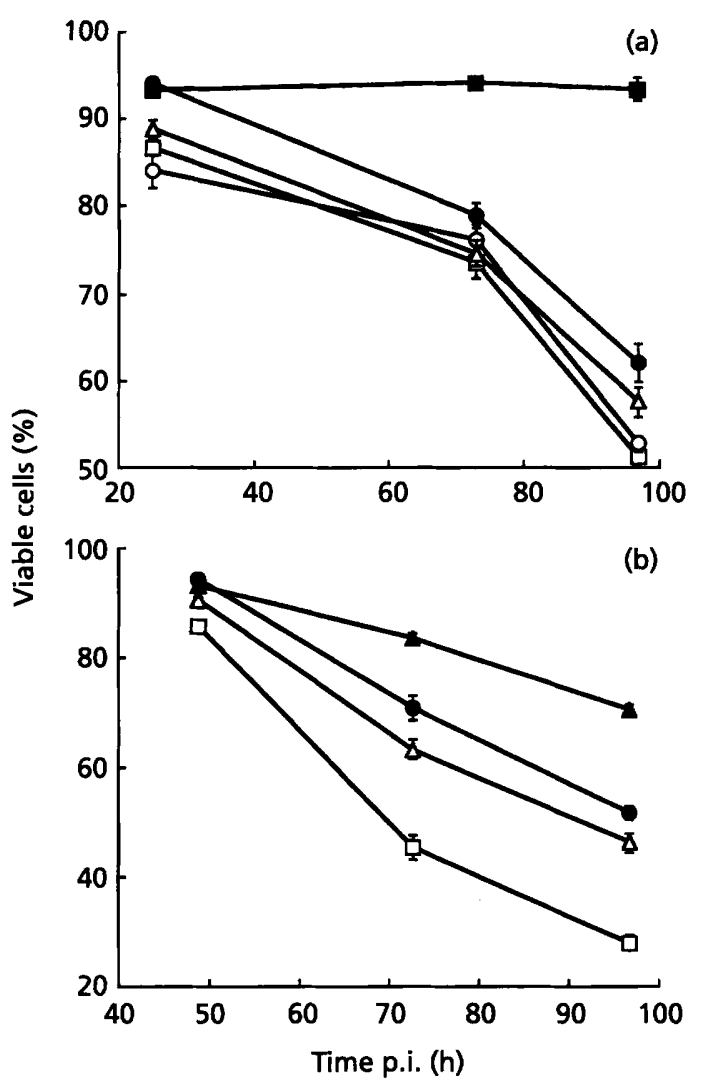

Fig. 4. Influence of $\alpha$-lipoic acid on the viability of the EA.hy 926 endothelial cells infected with $R$. rickettsii. (a) Overnight pretreatment with different concentrations of $\alpha$-lipoic acid followed by infection with $R$. rickettsii. (b) Supplementation with different concentrations of $\alpha$-lipoic acid p.i. with $R$. rickettsii. The number of viable cells was determined by the trypan blue dye exclusion test and expressed as the percentage of the total number of cells harvested per dish for each experimental variable $(n=4)$. $\square$, uninfected, untreated cells; $\square$, infected cells; $O$, cells treated with $10 \mu \mathrm{M} \alpha$-lipoic acid before or after infection; $\Delta$, cells treated with $100 \mu \mathrm{M} \alpha$-lipoic acid before or after infection; 0 , cells treated with $200 \mu \mathrm{M} \alpha$ lipoic acid before or after infection; $\boldsymbol{\Delta}$, cells treated with $500 \mu \mathrm{M} \alpha$-lipoic acid before or after infection. of EA.hy 926 endothelial cells resulted in a twofold increase in peroxide levels, while the levels of reduced glutathione and glutathione peroxidase decreased by half. Although very similar trends in the direction of changes of corresponding variables were demonstrated in HUVEC at $72 \mathrm{~h}$ p.i., the changes were not quantitatively identical since peroxide levels increased 1.3fold, while the amount of thiols and the activity of glutathione peroxidase decreased $2 \cdot 7$ - and $1 \cdot 8$-fold, respectively (Table 1 ).

In other experiments the time course of biochemical changes in EA.hy 926 endothelial cells, uninfected and infected with $R$. rickettsii, was determined. These results are given below as the baseline levels observed for experiments with $\alpha$-lipoic acid treatment.

\section{Influence of $\alpha$-lipoic acid on viability of infected EA.hy 926 cells}

Overnight pretreatment of uninfected EA.hy 926 cell cultures with 1,10,100 and $200 \mu \mathrm{M} \alpha$-lipoic acid did not prevent cells from being destroyed following $R$. rickettsii infection (Fig. 4a). At 96 h p.i., the percentage of viable cells in cultures pretreated with $\alpha$-lipoic acid varied from 49 to $61 \%$ compared with $50 \%$ in control untreated, infected samples. At $96 \mathrm{~h}$, the viability of cells pretreated with 100 and $200 \mu \mathrm{M} \alpha$-lipoic acid was significantly different from all other groups $(P<0.05)$, but the small differences observed may have been due to a small amount of carry-over of $\alpha$-lipoic acid since the dishes were not extensively rinsed.

However, when the culture medium was supplemented once with 100,200 and $500 \mu \mathrm{M} \alpha$-lipoic acid $2 \mathrm{~h}$ p.i. and after adherence of rickettsiae, it increased the viability of infected cells at $96 \mathrm{~h}$ to 45,51 and $70 \%$, respectively, compared with $26 \%$ viability of cells in untreated, infected cultures (Fig. 4b). At both 72 and $96 \mathrm{~h}$ p.i., viabilities at all concentrations were significantly different $(P<0.05)$. One and $10 \mu \mathrm{M} \alpha$-lipoic acid did not prevent cells from being destroyed by rickettsiae (data 


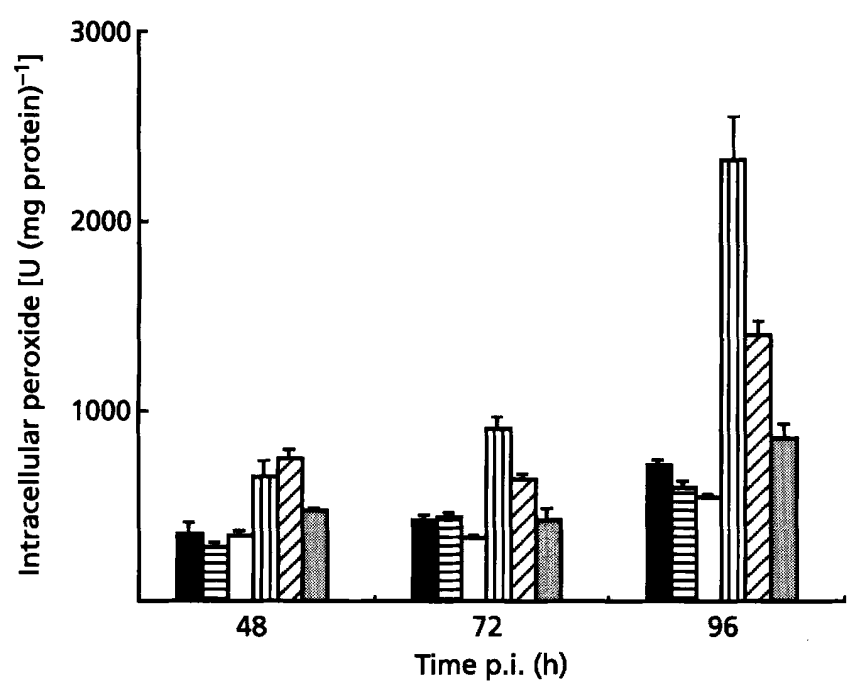

Fig. 5. Intracellular peroxide levels in uninfected EA.hy 926 endothelial cells untreated (control, $\square$ ) or treated with 100 (目) or $500 \mu \mathrm{M}(\square) \alpha$-lipoic acid and in EA.hy 926 cells infected with $R$. rickettsii and cultivated in medium without (四) and with 100 ( $\square)$ or $500 \mu \mathrm{M}$ (웡 $\alpha$-lipoic acid. The data are presented as mean \pm SEM.

not shown). The viability of control uninfected cells cultivated in the presence of $1,10,100,200$ or $500 \mu \mathrm{M} \alpha$ lipoic acid did not change over $96 \mathrm{~h}$ of experiments and was close to $90 \%$ as found for uninfected, untreated cultures (Fig. 4a).

\section{Influence of $\alpha$-lipoic acid supplementation on measurement of oxidative injury elicited by $R$. rickettsii infection of EA.hy $\mathbf{9 2 6}$ cells}

Cultivation of uninfected EA.hy 926 cells resulted in a slight increase in intracellular peroxide levels at $72(P<$ $0.05)$ and $96 \mathrm{~h}(P<0.01)$ p.i. (Fig. 5). Uninfected cells maintained in medium with $100 \mu \mathrm{M} \alpha$-lipoic acid displayed similar changes in peroxide levels, but supplementation with $500 \mu \mathrm{M} \alpha$-lipoic acid resulted in slight decreases in the amounts of peroxide, significant at $96 \mathrm{~h}$ $(P<0.001)$, when compared to untreated controls. Infection with $R$. rickettsii resulted in an increase of intracellular peroxide levels at 48 and $72 \mathrm{~h}$ p.i. and the peroxide level was particularly enhanced at $96 \mathrm{~h}$ p.i. (Fig. 5). Cultivation of rickettsiae-infected EA.hy 926 cells in the presence of $100 \mu \mathrm{M} \alpha$-lipoic acid resulted in a significant reduction of intracellular peroxide levels at 72 and $96 \mathrm{~h}$ p.i. compared with untreated, infected cells $(P<0.01)$. Effects of treatment with $500 \mu \mathrm{M} \alpha$-lipoic acid at 48,72 and $96 \mathrm{~h}$ p.i. were more pronounced and reduced intracellular peroxide levels in infected cells to the levels found in uninfected, untreated cells (Fig. 5).

Uninfected, untreated EA.hy 926 cells had significantly decreased reduced glutathione levels after 72 and $96 \mathrm{~h}$ of cultivation compared with those measured at $48 \mathrm{~h}$ p.i. $(P<0.05$, Fig. 6). Supplementation of culture medium with 100 and $500 \mu \mathrm{M} \alpha$-lipoic acid did not result in

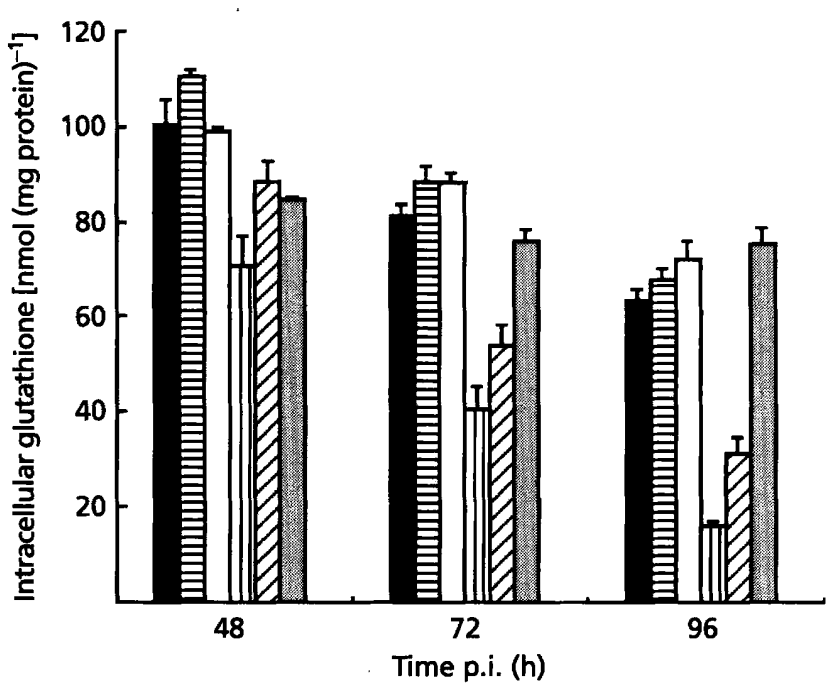

Fig. 6. Intracellular reduced glutathione levels in uninfected EA.hy 926 endothelial cells untreated (control, $\square$ ) or treated with 100 (目) or $500 \mu \mathrm{M}(\square) \alpha$-lipoic acid and in EA.hy 926 cells infected with $R$. rickettsii and cultivated in medium without (四) and with $100(\square)$ or $500 \mu \mathrm{M}$ (圈) $\alpha$-lipoic acid. The data are presented as mean \pm SEM.

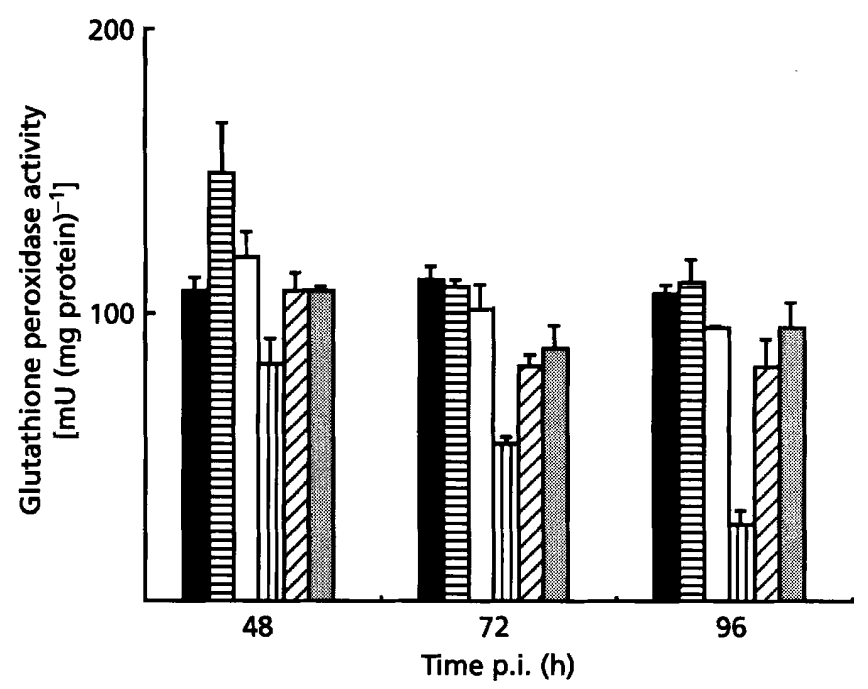

Fig. 7. Glutathione peroxidase activity in uninfected EA.hy 926 endothelial cells untreated (control, $\square$ ) or treated with 100 (目) or $500 \mu \mathrm{M}(\square) \alpha$-lipoic acid and in EA.hy 926 cells infected with $R$. rickettsii and cultivated in medium without (四) and with 100 (飞) or $500 \mu \mathrm{M}$ (圈) $\alpha$-lipoic acid. The data are presented as mean \pm SEM.

significant changes compared to untreated cells at all time points. $R$. rickettsii infection of untreated EA.hy 926 cells caused the significant depletion of intracellular reduced glutathione by $48 \mathrm{~h}$ p.i. and this effect was even more pronounced after 72 and $96 \mathrm{~h}(P<0.01$, Fig. 6). In contrast, infected EA.hy 926 cells cultivated in the presence of 100 and $500 \alpha$-lipoic acid had elevated levels of glutathione at 48,72 and $96 \mathrm{~h}$ p.i. over untreated, 
infected cells. This effect was especially marked after treatment with $500 \mu \mathrm{M} \alpha$-lipoic acid for 72 and $96 \mathrm{~h}$, which restored intracellular thiol levels to those of both uninfected, untreated and uninfected, treated cells $(P<$ 0.05 , Fig. 6)

Changes in glutathione peroxidase activity were not significant in both uninfected, untreated and uninfected, treated EA.hy 926 cells at 48,72 and 96 h p.i. (Fig. 7). Inoculation of untreated EA.hy 926 cells with $R$. rickettsii and incubation for 48,72 and $96 \mathrm{~h}$ resulted in significant decreases in enzyme activity $(P<0 \cdot 01)$ which paralleled the changes observed for reduced glutathione levels. Supplementation of the culture medium with 100 and $500 \mu \mathrm{M} \alpha$-lipoic acid resulted in significant restoration of glutathione peroxidase activity to levels close to that in uninfected, untreated and uninfected, treated cells (Fig. 7). The effects of treatment with 100 and $500 \mu \mathrm{M} \alpha$-lipoic acid were not significantly different.

\section{Transmission electron microscopy}

The ultrastructure of EA.hy 926 cells infected with $R$. rickettsii was examined at $72 \mathrm{~h}$ p.i. The appearance of control uninfected EA.hy 926 cells is shown in Fig. 8(a). Multiple endocytic vesicles were located in proximity to the internal side of the cellular membrane in the cytoplasm, confirming the endothelial nature of EA.hy 926 cells. The cytoplasm of EA.hy 926 cells also contained mitochondria located free or associated with membranes of the endoplasmic reticulum, smooth and rough endoplasmic reticulum (RER) with associated ribosomes and significant numbers of vesicular structures. EA.hy 926 cells infected for $72 \mathrm{~h}$ with $R$. rickettsii exhibited very characteristic changes in their cellular architecture (Fig. 8b). They exhibited dilatation of the RER and outer nuclear envelope (ONE), much as was previously described in HUVEC (Silverman, 1984). Distension of the RER and ONE resulted in a large intracellular cisterna that sometimes encompassed more than half of the cytoplasm of the infected cells. Dilated cisternae contained numerous host-cell membrane fragments or cytoplasmic islets, a fine granular substance, presumably intracisternal cell protein, and rickettsiae, free or bounded by membranes. Numerous rickettsiae of typical morphology were seen in the cytoplasm: they were either free in the cytosol or circumscribed by membranes of dilating RER. All rickettsiae were surrounded by the characteristic electron-lucent zone generally described as a microcapsule. Mitochondria located in the cytoplasm had swollen and sometimes disrupted cristae. In some cells, structures resembling condensed actin structures could be seen in the cytoplasm at the cell periphery.

Cultivation of infected EA.hy 926 cells in the presence of 100 and $200 \mu \mathrm{M} \alpha$-lipoic acid for $72 \mathrm{~h}$ significantly reduced but did not prevent development of pathological changes in cellular architecture. Some of the infected, treated cells continued to display the distension in the
ONE compartment and dilated areas contained clusters of membrane structures and rickettsiae (Fig. 8c). A small portion of the infected cells cultivated under these conditions contained no signs of membrane dilatation of the RER. Uninfected cells maintained in the medium with 100 and $200 \mu \mathrm{M} \alpha$-lipoic acid resembled untreated, uninfected cells in their structure (not shown).

The appearance of $R$. rickettsii-infected EA.hy 926 cells cultivated with $500 \mu \mathrm{M} \alpha$-lipoic acid more closely resembled that of uninfected cells, except for the presence of intracytoplasmic rickettsiae (Fig. 8d). The ultrastructure of uninfected cells maintained in culture medium supplemented with $500 \mu \mathrm{M} \alpha$-lipoic acid did not display any atypical changes.

\section{R. rickettsii plaque formation in the EA.hy 926 cell line}

A suspension of $R$. rickettsii containing $7 \cdot 01 \times 10^{8}$ p.f.u. $\mathrm{ml}^{-1}$ was titrated in EA.hy 926 cells. The ability of $R$. rickettsii to form plaques was examined for $10 \mathrm{~d}$ p.i. Diffuse areas of cytopathic changes were detected in wells inoculated with a $10^{-5}$ dilution and only a few lytic areas were seen at $10^{-6}$ dilution. Because of this, the number of plaques formed was difficult to estimate.

Application on day 2 p.i. of a secondary agarose overlay that contained emetine, an inhibitor of eukaryotic protein synthesis, and $\mathrm{NaF}$, an inhibitor of glycolysis, resulted in clearly distinguishable lytic plaques (Fig. 9). The appearance of plaques in EA.hy 926 cells differed from that of plaques produced in Vero cells by having a smaller size and less defined shape. The titre of $R$. rickettsii in EA.hy 926 cells was estimated to be $6.6 \times 10^{7} \pm 0.8 \times 10^{7}$, suggesting that plaquing efficiency was approximately $10 \%$ of that obtained with Vero cells.

\section{DISCUSSION}

The permanent endothelial cell line EA.hy 926 has been characterized in many preliminary studies which demonstrated that these hybrid cells express many highly differentiated functions that are characteristic of vascular endothelium. For example, EA.hy 926 cells contain the Weibel-Palade bodies (Edgell et al., 1990), specialized storage vesicles characteristic of endothelial cells. EA.hy 926 cells also possess stable endothelial characteristics during in vitro cultivation, including fibrinolytic properties (Emeis \& Edgell, 1988), expression and morphological distribution of factor VIII-related antigen (Edgell et al., 1983) and the endothelial adhesion molecules, ICAM-1 and VCAM-1 (Thornhill et al., 1993), and expression and secretion of endothelin-1 (Saijonmaa et al., 1991) and prostacyclin (Suggs et al., 1986) that are the same as found in primary endothelial cells.

The response of the endothelial cell line EA.hy 926 to infection with $R$. rickettsii was demonstrated in this 

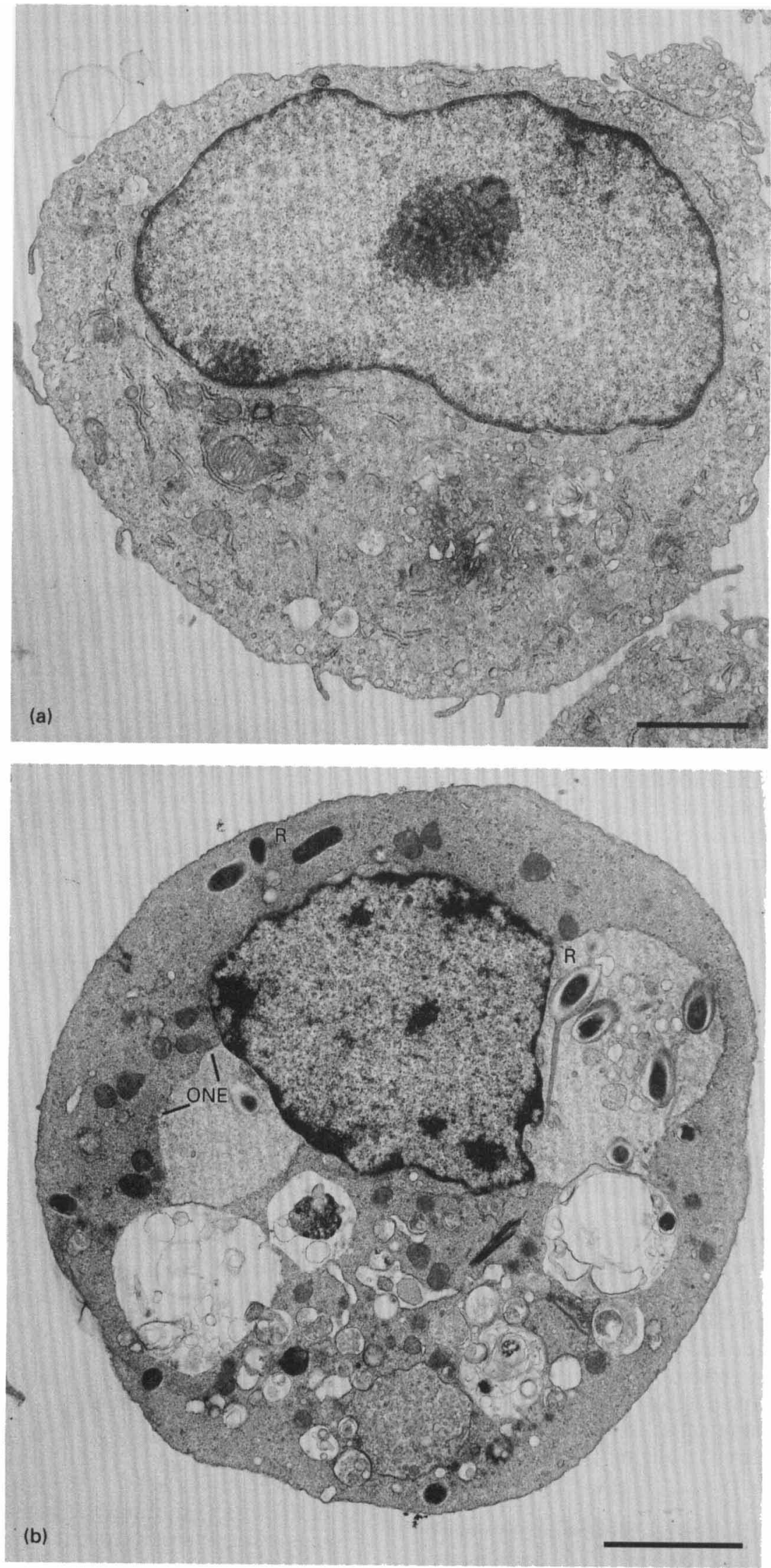

Fig. 8. Transmission electron micrograph of uninfected and $R$. rickettsii-infected EA.hy 926 endothelial cells from a $72 \mathrm{~h}$ p.i. culture. (a) Uninfected cell. (b) Cell infected with $R$. rickettsii, demonstrating extensive dilatation of membranes of 

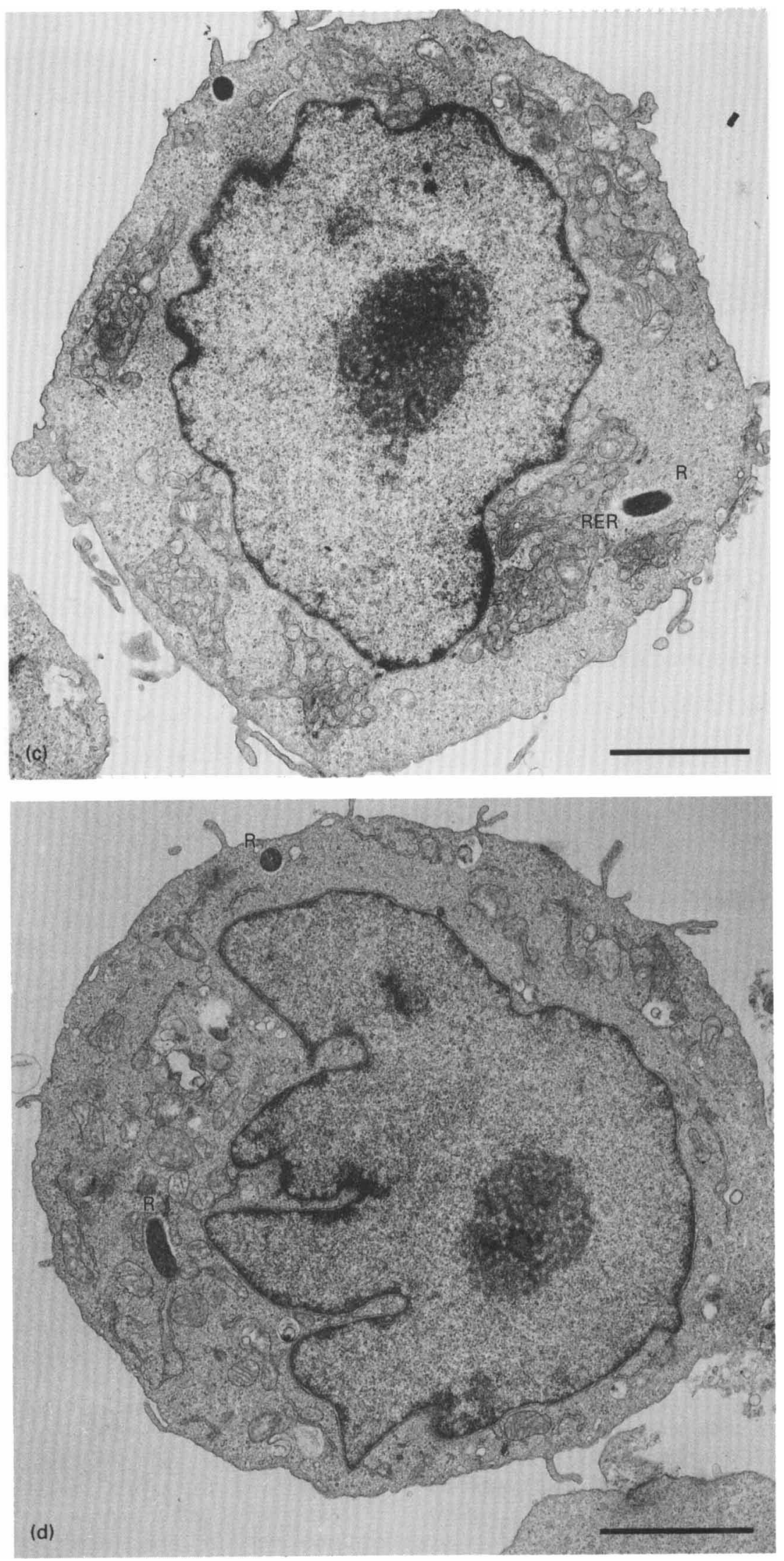

the ONE and intracellular rickettsiae (R). (c) Infected cell cultivated in medium supplemented with $200 \mu \mathrm{M} \alpha$-lipoic acid in an early stage of membrane dilatation. (d) Infected cell cultivated in medium supplemented with $500 \mu \mathrm{M} \alpha$-lipoic acid. Bars, $2 \mu \mathrm{m}$. 
(a)

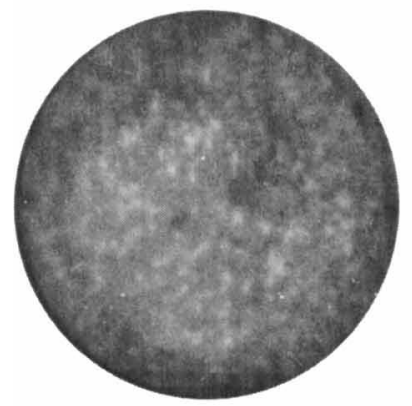

$10^{-5}$ (b)

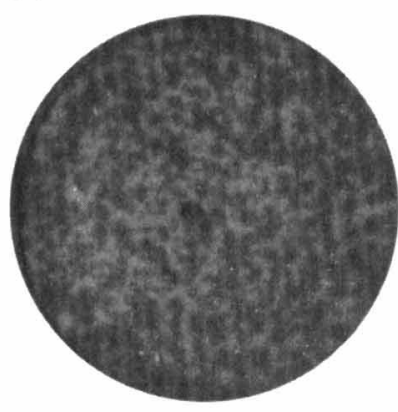

$10^{-5}$

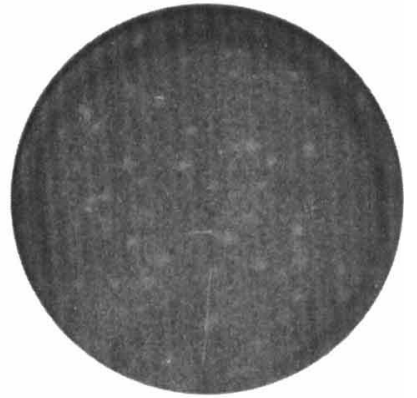

$10^{-6}$

Fig. 9. R. rickettsii plaque formation in EA.hy 926 endothelial cells. (a) Plaque formation in untreated EA.hy 926 cells. (b) Plaque formation in the presence of $2 \mu \mathrm{g}$ emetine $\mathrm{ml}^{-1}$ and $40 \mu \mathrm{g} \mathrm{NaF} \mathrm{ml} \mathrm{m}^{-1}$. Plaques were detected and counted $10 \mathrm{~d}$ p.i. following staining with $0.3 \%$ neutral red for $2 \mathrm{~h}$. Numbers below the plates indicate the dilution of the inoculum.

study. Infection resulted in normal intracellular multiplication of rickettsiae. Rickettsial growth had similar kinetics to that previously found in HUVEC and rickettsiae catalysed the formation of actin tails that are associated with cell-to-cell spread (Heinzen et al., 1993).

R. rickettsii infected the EA.hy 926 cells and caused a cytotoxic effect. This was demonstrated by the development of specific rickettsial lytic plaques in EA.hy 926 cell monolayers. The efficiency of plaquing of $R$. rickettsii on EA.hy 926 cells was approximately 10 times lower than that obtained in parallel experiments with Vero cells. A similar lower plaquing efficiency for HUVEC compared to that obtained with Vero cells and primary chicken fibroblasts was determined by Walker et al. (1982). These data suggest that there are differences in the efficiency of rickettsial adhesion, intracellular replication and cell-to-cell spread in cells of different origin. This difference is likely to be related to specific expression of an as yet unidentified host-cell factor recognized by rickettsiae. Differences in the composition of the cell culture medium required for growth of individual cell lines, unique cofactors and medium supplements are other important factors which may influence the character of $R$. rickettsii infection (Walker et al., 1982).

Intracellular multiplication of $R$. rickettsii caused significant changes in the biochemical properties of EA.hy 926 cells, as shown here for the antioxidant system. Infection resulted in increased levels of intracellular peroxides and decreased levels of intracellular reduced glutathione and glutathione peroxidase activity. Similar changes have been described previously in primary HUVEC (Devamanoharan et al., 1994; Silverman \& Santucci, 1988, 1990) and as we confirmed here in our control experiments with HUVEC (Table 1). These findings suggest that pathophysiological events occurring in the two endothelial cell lines have similar mechanisms. Development of oxidative changes following $R$. rickettsii infection correlated with a consistent appearance of dilated membranes of the endoplasmic reticulum and ONE. The type of ultrastructural reorganization undergone by EA.hy 926 cells infected with $R$. rickettsii resembled remarkably the type of rickettsial injury which occurs in infected HUVEC (Silverman, 1984). Common mechanisms are probably involved in the development of injury following rickettsial infection in the two endothelial cell types.

Although a direct toxic action of rickettsiae cannot be ruled out completely, oxidative injury and associated lipid peroxidation has been suggested as a potential mechanism for explaining the destruction of HUVEC following infection with $R$. rickettsii (Silverman \& Santucci, 1988). The generation of free radicals as the result of cellular metabolism causes peroxidation of lipids of intracellular membranes and the subsequent damage can result in abnormal membrane permeability. The influx of water and sodium ions through the damaged membranes may then cause dilatation of the intracisternal space. If this hypothesis is correct, the administration of antioxidants should protect endothelial cells against $R$. rickettsii-associated injury. $\alpha$ Lipoic acid, a metabolic antioxidant with a very wide spectrum of activity, is easily absorbed from the diet and is converted intracellularly to its reduced form, dihydrolipoic acid (Packer et al., 1995). The former provides noticeable protection against lipid peroxidation by converting oxidized intracellular glutathione to its reduced form (Bast \& Haenen, 1988). Consequently, the treatment of EA.hy 926 endothelial cells with the antioxidant $\alpha$-lipoic acid was evaluated for comparison with studies performed previously with HUVEC (Eremeeva \& Silverman, 1998). We determined that supplementation of cell culture medium with 100 and $500 \mu \mathrm{M} \alpha$-lipoic acid protects EA.hy 926 cells against injury associated with $R$. rickettsii infection. Protection was apparent as an increase in the longevity of infected monolayers and resistance to cytopathic effects due to rickettsiae. Treatment with 100 and $200 \mu \mathrm{M} \alpha$-lipoic acid delayed and treatment with $500 \mu \mathrm{M} \alpha$-lipoic acid prevented development of pathological morphological changes in EA.hy 926 cells following $R$. rickettsii 
infection for at least 72 h. $\alpha$-Lipoic acid also increased intracellular levels of reduced glutathione and glutathione peroxidase activity and decreased intracellular peroxide levels in infected EA.hy 926 cells, sometimes to levels found in uninfected, untreated cells. These effects of $\alpha$-lipoic acid on $R$. rickettsii-infected EA.hy 926 cells were very similar to those previously demonstrated for infected HUVEC (Eremeeva \& Silverman, 1998). However, we did not observe an increase in intracellular thiol levels in uninfected EA.hy 926 cells maintained in medium with $\alpha$-lipoic acid for 96 h p.i. Similar treatment of uninfected HUVEC caused a dose-dependent elevation of intracellular reduced glutathione. The protective role of intracellular thiol pools against $R$. rickettsii-induced injury in HUVEC has been suggested previously (Silverman \& Santucci, 1990). However, protection conferred by $\alpha$-lipoic acid on EA.hy 926 cells and HUVEC against $R$. rickettsii-induced injury may be due to different primary antioxidant effects of this compound in the two endothelial cell lines (direct scavenging of reactive oxygen species, increase of intracellular thiol pool and/or regeneration of intracellular antioxidant pools) (Packer et al., 1995). Uninfected HUVEC also responded at $96 \mathrm{~h}$ p.i. to incubation with $500 \mu \mathrm{M} \alpha$-lipoic acid by showing a significant decline in viability and changes in cell shape. In contrast, the uninfected EA.hy 926 cells, untreated or treated with $\alpha$-lipoic acid, maintained their viability at the $90 \%$ level for $96 \mathrm{~h}$ and no cell shape changes were observed. Again these results suggest that the two types of endothelial cells have somewhat different responses to identical $\alpha$-lipoic acid treatments. These may be related to obvious differences in cellular metabolism between primary HUVEC, which are poorly adapted to growth under in vitro conditions (Gimbrone, 1976) and the permanent endothelial EA.hy 926 cells. The latter are able to sustain unlimited growth (Edgell et al., 1983), possibly due to alteration of restrictive endothelial cell regulatory mechanisms by genes from the tumour cell fusion partner.

Interestingly, another difference between HUVEC and EA.hy 926 cells toward $R$. rickettsii infection was that the total yield of viable micro-organisms from the EA.hy 926 cells was lower than that with HUVEC. As with the variable effects of $\alpha$-lipoic acid, these results may reflect the genetically determined heterogeneity of different batches of HUVEC originating from humans with different genetic backgrounds and possessing variable susceptibility to $R$. rickettsii infection. Individuals with lowered levels of glucose-6-phosphate dehydrogenase develop more severe forms of Rocky Mountain spotted fever (Walker et al., 1981). Humans are also heterogeneous in their level of expression and isoenzymes of several other enzymes, such as catalase (Shingu et al., 1985; Ogata, 1991), superoxide dismutase (Sandstrom et al., 1994) and glutathione peroxidase (Spector et al., 1996). Since the activities of these enzymes are altered during in vitro infection of HUVEC (Devamanoharan et al., 1994; Santucci et al., 1992), different levels of intrinsic expression of catalase, superoxide dismutase and glutathione peroxidase may also influence cell susceptibility to $R$. rickettsii in vitro and in vivo and affect the severity of acquired disease. This suggestion could be tested by the preparation of primary endothelial cell cultures of known genetic types from individual cords and comparison of their responses to $R$. rickettsii infection.

In summary, the permanent EA.hy 926 cell line can be used as a homogeneous experimental cell line which permits more consistent responses to specific variables and greater reproducibility of data. However, it may also be valuable to compare the characteristics of $R$. rickettsii infection in the HMEC-1 permanent endothelial cell line derived from the microvascular endothelium (Ribeiro et al., 1995), since it may be an even more appropriate model for studies of rickettsia-induced cell injury than cells originating from umbilical veins.

\section{ACKNOWLEDGEMENTS}

This work was supported by Public Health Service grant AI 17416 from the National Institute of Allergy and Infectious Diseases. The authors are greatly thankful to Dr Cora-Jean S. Edgell for the gift of EA.hy 926 endothelial cell line, to $\mathrm{Dr}$ Gregory A. Dasch for his review of the manuscript and gift of anti- $R$. rickettsii serum, to Lisa Santucci for her assistance with biochemical procedures, to $\mathrm{Dr}$ Xiaojiang Tian for preparation of primary cultures of human endothelial cells and to Perry Comegys for excellent photographic work.

\section{REFERENCES}

Aniskovich, L. P., Eremeeva, M. E., Balayeva, N. M., Ignatovich, V. F., Artemiev, M. I., Emelianov, V. V. \& Smirnova, N. S. (1989). Methods for purification of Rickettsia prowazekii separated from host tissue: a step-by-step comparison. Acta Virol 33, 361-370.

Bast, A. \& Haenen, G. R. M. M. (1988). Interplay between lipoic acid and glutathione in the protection against microsomal lipid peroxidation. Biochim Biophys Acta 963, 558-561.

Cathcart, R., Schwiers, E. \& Ames, B. N. (1983). Detection of picomole levels of hydroperoxides using a fluorescent dichlorofluorescein assay. Anal Biochem 134, 111-116.

Clerc, P. \& Sansonetti, P. J. (1987). Entry of Shigella flexneri into HeLa cells: evidence for directed phagocytosis involving actin polymerization and myosin accumulation. Infect Immun 55, 2681-2688.

Devamanoharan, P. S., Santucci, L. A., Hong, J. E., Tian, X. \& Silverman, D. J. (1994). Infection of human endothelial cells by Rickettsia rickettsii causes a significant reduction in the levels of key enzymes involved in protection against oxidative injury. Infect Immun 60, 5113-5118.

Edgell, C.-J. S., McDonald, C. C. \& Graham, J. B. (1983). Permanent cell line expressing human factor VIII-related antigen established by hybridization. Proc Natl Acad Sci USA 80, 3734-3737.

Edgell, C.-J. S., Haizlip, J. E., Bagnell, C. R., Packenham, J. P., Harrison, P., Wilbourn, B. \& Madden, V. J. (1990). Endothelium specific Weibel-Palade bodies in a continuous human cell line, EA.hy 926. In Vitro Cell Dev Biol 26, 1167-1172. 
Emeis, J. J. \& Edgell, C.-J. S. (1988). Fibrinolytic properties of a human endothelial hybrid cell line (EA.hy 926). Blood 71, 1669-1675.

Eremeeva, M. E. \& Silverman, D. J. (1998). Effects of the antioxidant $\alpha$-lipoic acid on human umbilical vein endothelial cells infected with Rickettsia rickettsii. Infect Immun 66, 2290-2299.

Eremeeva, M. E., Balayeva, N. M., Ignatovich, V. F. \& Raoult, D. (1993). Proteinic and genomic identification of spotted fever group rickettsiae isolated in the former USSR. J Clin Microbiol 31, 2625-2633.

Gimbrone, M. A. (1976). Culture of vascular endothelium. Prog Hemostasis Thromb 3, 2745-2756.

Gimenez, D. F. (1964). Staining rickettsiae in yolk-sac cultures. Stain Technol 39, 135-140.

Heinzen, R. A., Hayes, S. F., Peacock, M. G. \& Hackstadt, T. (1993). Directional actin polymerization associated with spotted fever group rickettsia infection of Vero cells. Infect Immun 61, 1926-1935.

Lauer, B. A., Reller, L. B. \& Mirrett, S. (1981). Comparison of acridine orange and Gram stains for detection of microorganisms in cerebrospinal fluid and other clinical specimens. $J$ Clin Microbiol 14, 201-205.

Ogata, M. (1991). Acatalasemia. Hum Genet 86, 331-340.

Packer, L., Witt, E. H. \& Tritschler, H. J. (1995). Alpha-lipoic acid as a biological antioxidant. Free Radic Biol Med 19, 227-250.

Paglia, D. E. \& Valentine, W. N. (1967). Studies on the quantitative and qualitative characterization of erythrocyte glutathione peroxidase. J Lab Clin Med 70, 158-169.

Policastro, P. F., Peacock, M. G. \& Hackstadt, T. (1996). Improved plaque assays for Rickettsia prowazekii in Vero 76 cells. J Clin Microbiol 34, $1944-1948$.

Ribeiro, M. J. A., Phillips, D. J., Benson, J. M., Evatt, B. L., Ades, E. W. \& Hooper, W. C. (1995). Hemostatic properties of the SV-40 transfected human microvascular endothelial cell line (HMEC-1). Thromb Res 79, 153-161.

Saijonmaa, O., Nyman, T., Hohenthal, U. \& Fyhrquist, F. (1991). Endothelin-1 expressed and released by a human endothelial hybrid cell line (EA.hy 926). Biochem Biophys Res Commun 181, 529-536.

Sandstrom, J., Nilsson, P., Karlsson, K. \& Marklund, S. L. (1994). 10 -fold increase in human plasma superoxide dismutase content caused by a mutation in heparin-binding domain. $J$ Biol Chem 269, 19163-19166.

Santucci, L. A., Gutierrez, P. L. \& Silverman, D. J. (1992). Rickettsia rickettsii induces superoxide radical and superoxide dismutase in human endothelial cells. Infect Immun 60, 5113-5118.

Saville, B. (1958). A scheme for the colorimetric determination of microgram amounts of thiols. Analyst 83, 670-672.
Shingu, M., Yoshioka, K., Nobunaga, M. \& Yoshida, K. (1985). Human vascular smooth muscle cells and endothelial cells lack catalase activity and are susceptible to hydrogen peroxide. Inflammation 9, 309-320.

Silverman, D. J. (1984). Rickettsia rickettsii-induced cellular injury of human vascular endothelium in vitro. Infect Immun 44, $545-553$.

Silverman, D. J. \& Santucci, L. A. (1988). Potential for free radicalinduced lipid peroxidation as a cause of endothelial cell injury in Rocky Mountain spotted fever. Infect Immun 56, 3110-3115.

Silverman, D. J. \& Santucci, L. A. (1990). A potential protective role for thiols against injury caused by Rickettsia rickettsii. Ann N Y Acad Sci 590, 111-117.

Silverman, D. J., Santucci, L. A. \& Sekeyova, Z. (1991). Heparin protects human endothelial cells infected by Rickettsia rickettsii. Infect Immun 59, 4505-4510.

Smith, P. K., Krohn, R. I., Hermanson, G. T., Mallia, A. K., Gartner, F. H., Provenzano, M. D., Fujimoto, E. K., Goeke, N. M., Olson, B. J. \& Klenk, D. C. (1985). Measurement of protein using bicinchoninic acid. Anal Biochem 150, 76-85.

Spector, A., Yang, Y., Ho, Y.-S., Magnenat, J.-L., Wang, R.-R., Ma, W. \& Li, W.-C. (1996). Variation in cellular glutathione peroxidase activity in lens epithelial cells, transgenics and knockouts does not significantly change the response to $\mathrm{H}_{2} \mathrm{O}_{2}$ stress. Exp Eye Res 62, 521-540.

Suggs, J. E., Madden, M. C., Friedman, M. \& Edgell, C.-J. S. (1986). Prostacyclin expression by a continuous human cell line derived from vascular endothelium. Blood 68, 825-829.

Thornhill, M. H., Li, J. \& Haskard, D. O. (1993). Leucocyte endothelial cell adhesion: a study comparing human umbilical vein endothelial cells and the endothelial cell line EA-hy-926. Scand J Immunol 38, 279-286.

Walker, D. H. (1988). Pathology and pathogenesis of the vasculotropic rickettsioses. In Biology of Rickettsial Diseases, vol. 1, pp. 115-138. Edited by D. H. Walker. Boca Raton, FL: CRC Press.

Walker, D. H., Kirkman, H. N. \& Wittenberg, P. H. (1981). Genetic states possibly associated with enhanced severity of Rocky Mountain spotted fever. In Rickettsiae and Rickettsial Diseases, pp. 621-630. Edited by W. Burgdorfer \& R. L. Anacker. New York: Academic Press.

Walker, D. H., Firth, W. T. \& Edgell, C.-J.S. (1982). Human endothelial cell culture plaques induced by Rickettsia rickettsii. Infect Immun 37, 301-306.

Wike, D. A. \& Burgdorfer, W. (1972). Plaque formation in tissue culture by Rickettsia rickettsii isolated directly from whole blood and tick hemolymph. Infect Immun 6, 736-738.

Received 3 February 1998; revised 15 April 1998; accepted 22 April 1998. 